\title{
Advanced platelet-rich fibrin promotes the paracrine function and proliferation of adipose-derived stem cells and contributes to micro-autologous fat transplantation by modulating HIF-1 $\alpha$ and VEGF
}

\author{
Hao Huang ${ }^{1 \#}$, Xike Tang ${ }^{2 \#}$, Shounan $\mathrm{Li}^{3}$, Donglin Huang ${ }^{4}$, Dongchangcheng $\mathrm{Lu}^{5}$, Fuzhi Wu ${ }^{5}$, Dalie Liu ${ }^{1}$, \\ Hongmian $\mathrm{Li}^{6}$
}

${ }^{1}$ Department of Plastic and Aesthetic Surgery, Zhujiang Hospital, Southern Medical University/The Second School of Clinical Medicine, Southern Medical University, Guangzhou, China; ${ }^{2}$ Dermatology Department, The Affiliated Nanning Infectious Disease Hospital of Guangxi Medical University and the Fourth People's Hospital of Nanning, Nanning, China; ${ }^{3}$ Department of Thoracic Surgery, the People's Hospital of Binyang County, Nanning, China; ${ }^{4}$ Department of Plastic and Aesthetic Surgery, The Fifth Affiliated Hospital of Guangxi Medical University \& The First People's Hospital of Nanning, Nanning, China; ${ }^{5}$ Nanning Wilking Biological Technology Co., Ltd., Nanning, China; ${ }^{6}$ Research Center of Medical Sciences, The People's Hospital of Guangxi Zhuang Autonomous Region \& Guangxi Academy of Medical Sciences, Nanning, China Contributions: (I) Conception and design: H Huang, S Li; (II) Administrative support: X Tang, D Huang; (III) Provision of study materials or patients: D Lu; (IV) Collection and assembly of data: D Lu, F Wu, X Tang; (V) Data analysis and interpretation: D Huang, D Liu, H Li; (VI) Manuscript writing: All authors; (VII) Final approval of manuscript: All authors.

\#These authors contributed equally to this work and should be considered as co-first author.

Correspondence to: Doctor Dalie Liu. Department of Plastic and Aesthetic Surgery, Zhujiang Hospital, Southern Medical University/The Second School of Clinical Medicine, Southern Medical University, Guangzhou 510282, China. Email: liudalie1955@163.com; Doctor Hongmian Li. Research Center of Medical Sciences, The People’s Hospital of Guangxi Zhuang Autonomous Region \& Guangxi Academy of Medical Sciences, Nanning 530021, China. Email: lihongmian@gxmu.edu.cn.

Background: The micro-autologous fat transplantation (MAFT) technique has demonstrated its feasibility in multiple medical fields, such as facial rejuvenation. Advanced platelet-rich fibrin $(A P R F)$, an autologous platelet concentrated on a fibrin membrane without added external factors, has shown significant potential for tissue restoration. However, the role of $A P R F$ in the modulation of MAFT remains unclear. Here, we aimed to explore the effect of $A P R F$ on MAFT.

Methods: Adipose-derived stem cells (ASCs) were isolated from human gastric subcutaneous fat and treated with APRF. ELISA assays measured cytokines. The proliferation of ASCs was analyzed by CCK-8 assays. The levels of hypoxia-inducible factor-1 $\alpha(H I F-1 \alpha)$, heat shock protein 70 (HSP70), insulin like growth factor 2 $(I G F-2)$, interleukin-6 (IL-6), interleukin-8 (IL-8), and vascular endothelial growth factor $(V E G F)$ were measured by ELISA assays, quantitative reverse transcription-PCR (qRT-PCR), and Western blot analysis. The effect of $A P R F / H I F-1 \alpha / V E G F$ on MAFT in vivo was analyzed in Balb/c nude mice. The BALB/c mice were subcutaneously co-transplanted with fat, $A P R F$, and control shRNA, HIF- $1 \alpha$ shRNA, or $V E G F$ shRNA into the dorsal area. The serum and protein levels of the above cytokines were analyzed by ELISA assays and Western blot analysis. Lipid accumulation was measured by Oil Red O staining. The expression of CD34 was assessed by immunohistochemical staining.

Results: $A P R F$ continuously secreted multiple cytokines, including epidermal growth factor (EGF), FGF-2, insulin like growth factor $1(I G F-1)$, interleukin-1beta $(I L-1 \beta)$, interleukin-4 $(I L-4)$, platelet-derived growth factor alpha polypeptide b $(P D G F-A B)$, platelet-derived growth factor beta polypeptide b $(P D G F-B B)$, transforming growth factor-beta $(T G F-\beta)$, and $V E G F$. APRF was able to promote the proliferation of ASCs. $A P R F$ dose-dependently activated the expression of HIF-1 $\alpha, H S P 70, I G F-2, I L-6, I L-8$, and $V E G F$ in ASCs. $A P R F$ regulated the paracrine function of ASCs by modulating HIF-1 $\alpha$ and VEGF. APRF increased the survival of MAFT by modulating HIF-1 $\alpha$ and VEGF in vivo. 
Conclusions: $A P R F$ promotes the paracrine function and proliferation of ASCs and contributes to MAFT by modulating $H I F-1 \alpha$ and $V E G F$. Our findings provide new insights into the mechanism by which $A P R F$ regulates MAFT.

Keywords: Micro-autologous fat transplantation (MAFT); adipose-derived stem cells (ASCs); paracrine; advanced platelet-rich fibrin $(A P R F)$; hypoxia-inducible factor-1 $\alpha(H I F-1 \alpha)$; vascular endothelial growth factor $(V E G F)$

Submitted Nov 12, 2021. Accepted for publication Jan 05, 2022.

doi: 10.21037/atm-21-6812

View this article at: https://dx.doi.org/10.21037/atm-21-6812

\section{Introduction}

Fat is a repeatedly accessible, host-compatible, readily available, abundant, inexpensive, and autologous filler that restores volume (1). Fat generally holds stem cells, and fat grafting potentially increases the character of the overlying skin and restores skin injuries, thereby restoring the face. Fat is regarded as preferable to temporary conventional fillers (2). A significant limitation of fat transplantation is the uncertainty of surgeons concerning its application, owing to the changeable and variable incidence of graft survival (3). Due to better knowledge of adipose tissue elements developed over the last decade, operators can ultimately predict each segment's function to adjust procedures, which increases the yield of targeted cells and enhances clinical outcomes (4). Unpredictable retention rates and morbidities of fat grafting have been reported, such as neurovascular injury, abscess, nodulation, and cyst formation (5). Structural fat grafting has gained widespread attention and has exhibited satisfactory clinical outcomes (6). The traditional surgical excision of fat mass or dermal fat mass transplantation has significant trauma, large surgical scar and may cause the defect of the donor area. microautologous fat transplantation (MAFT) can reduce the transplantation trauma, and there is no noticeable scar in the donor and recipient area. MAFT will make the shape of the receiving area uniform and natural, without body surface shadow and easy to shape (7-9). The theory of MAFT has been proposed, and the reliability of nodulation in facial rejuvenation has been validated $(6,10-12)$. However, it was found that the graft survival rate of simple transplantation of granular adipocytes was poor. Tang et al found that the adipose stem cells can significantly increase the expression of basic fibroblast growth factor, improve the microcirculation of grafts, and significantly improve the morphology and function of granulosa adipocytes (13). However, the modulation mechanism of MAFT is still poorly understood.

It has been shown that advanced platelet-rich fibrin $(A P R F)$, a concentrate of autologous platelets on a fibrin membrane without added external factors, presents significant potential for tissue restoration (14). APRF affects soft and bone tissue regeneration by the presence of monocytes/macrophages and their respective growth factors (15-17). The abundant platelets contained in APRF progressively release a high concentration of cytokines and growth factors, including vascular endothelial growth factor $(V E G F)$, insulin-like growth factor-1 $(I G F-1)$, and platelet-derived growth factor (PDGF), contributing to fat tissue engineering (18). However, the role of $A P R F$ in the modulation of MAFT remains elusive.

Hypoxia is described as a mismatch of cellular oxygen demand and supply. It is a feature of multiple diseases due to high proliferative rates, increased metabolism, and poor vascularization (19). The response to hypoxia is mainly mediated by the stabilization of hypoxia-related genes, such as hypoxia-inducible factor- $1 \alpha(H I F-1 \alpha)$, which transcriptionally activates over 300 genes (20). It has been reported that HIF signalling is involved in the modulation of fat transplantation (21), and VEGF contributes to fat transplantation (22). However, the correlation of these factors with $A P R F$ in the regulation of MAFT is still unclear.

In this study, we aimed to explore the role and underlying mechanism of $A P R F$ in manipulating MAFT. We identified a novel function of $A P R F$ in contributing to MAFT by regulating $H I F-1 \alpha$ and $V E G F$.

We present the following article following the ARRIVE reporting checklist (available at https://atm.amegroups. com/article/view/10.21037/atm-21-6812/rc). 


\section{Methods}

\section{Extraction and collection of APRF}

We selected three healthy adult volunteers $(20 \mathrm{~mL}$ per volunteer) venous blood was collected. After blood collection, it was put into 4 glass coated sterile negative pressure plastic tubes without anticoagulants. After centrifugation at 1,500 rpm for 14 minutes and rest for 5 minutes, we obtained 3 separation layers in the test tube. Discard the supernatant and cut off the longitudinal red end of the APRF layer to ensure that all red blood cell components were completely removed. Then, the APRF The extract of $(A P R F \mathrm{e})$ was stored at $4{ }^{\circ} \mathrm{C}$. The study was conducted in accordance with the Declaration of Helsinki (as revised in 2013). The present study was approved by the Institutional Review Board of the Fifth Affiliated Hospital of Guangxi Medical University (Nanning, Guangxi, China) (No. 2019-107-01). Written informed consent was provided by the donor patients.

\section{ASC isolation, culture, and identification}

Human gastric subcutaneous fat was obtained from excess tissues removed during reconstructive and plastic surgery. ASCs were isolated as previously reported (23-25). Briefly, adipose tissues were washed with phosphate-buffered saline (PBS) 3 times, cut into small pieces, and the extracellular matrixes were digested using collagenase solution $(0.1 \%)$ with shaking $\left(40\right.$ minutes, $\left.37^{\circ} \mathrm{C}\right)$. After adding Dulbecco's Modified Eagle Medium (DMEM) containing 1\% penicillin and $10 \%$ fetal bovine serum (FBS), the cell pellets were centrifuged ( 3 minutes, 1,600 rpm). The debris was removed, and the cells were cultured. The cells were cultured in DMEM (Gibco, Carlsbad, California, USA) containing 10\% FBS (Gibco, Carlsbad, California, USA), $0.1 \mathrm{mg} / \mathrm{mL}$ streptomycin (Gibco, Carlsbad, California, USA), and 100 units $/ \mathrm{mL}$ penicillin (Gibco, Carlsbad, California, USA) at $37^{\circ} \mathrm{C}$ with $5 \% \mathrm{CO}_{2}$. The Ethics Review Board of Zhujiang Hospital, Southern Medical University/ The Second School of Clinical Medicine, Southern Medical University approved the investigation concerning human participants. All cases provided written consent to participate in the research. APRF was obtained from Sangon Biotech (Sangon Biotech, Shanghai, China). HIF-1a shRNA, VEGF shRNA, and control shRNA were obtained from GenePharma (Shanghai, China).

We isolated adipose-derived stem cells (ASCs) from adipose tissue samples obtained from 1 healthy female volunteer who underwent abdominal liposuction
(24 years old, $163 \mathrm{~cm}, 58 \mathrm{~kg}$ ). Adipose tissue was digested with type I collagenase and P0 cells were extracted. To assess the ability of cells to differentiate into multiple lineages, we cultured p3-hasc in DMEM for 2-3 weeks. After that, we performed flow cytometry. The levels of cytokines secreted from $A P R F$ were analyzed by ELISA assays using the human epidermal growth factor $(E G F)$ ELISA Kit (Elabscience, Shanghai, China), Human bEGF ELISA Kit (Elabscience, Shanghai, China), human IGF-1 ELISA Kit (Boster, Wuhan, Hubei, China), human interleukin1beta $(I L-1 \beta)$ ELISA Kit (Elabscience, Shanghai, China), human interleukin-4 (IL-4) ELISA Kit (Elabscience, Shanghai, China), human platelet-derived growth factor alpha polypeptide b (PDGF-AB) ELISA Kit (Elabscience, Shanghai, China), human platelet-derived growth factor beta polypeptide b (PDGF-BB) ELISA Kit (Elabscience, Shanghai, China), human transforming growth factor-beta (TGF- $\beta$ ) ELISA Kit (Mlbio, Shanghai, China), and human VEGF ELISA Kit (Mlbio, Shanghai, China).

\section{Effect of APRF on ASC proliferation and paracrine function in vitro}

ASCs were harvested at the third passage and cultured in 96-well plates at a concentration of $2 \times 10^{4}$ cells per well in a growth culture medium ( 24 wells per group). 24 hours later, cells were treated for up to 7 days with basal media (BM, control) or 1 of the following 5 types of $A P R F$ media: BM plus $1.25 \% A P R F, \mathrm{BM}$ plus $2.5 \% A P R F, \mathrm{BM}$ plus $5 \% A P R F, \mathrm{BM}$ plus $10 \% A P R F$, and $\mathrm{BM}$ plus $20 \% A P R F$. The proliferation of ASCs was assessed using the cell counting kit-8 (CCK-8) assay (Kumamoto, Japan) and absorbance was measured at $450 \mathrm{~nm}$ using a microplate scanning spectrophotometer (ELx800, BioTek). The levels of cytokines in the culture medium were analyzed by ELISA assays, including the HIF-1 $\alpha$ ELISA Kit (Elabscience, Shanghai, China), heat shock protein 70 (HSP70) ELISA Kit (Elabscience, Shanghai, China), Human IGF-2 ELISA Kit (Boster, Wuhan, Hubei, China), Human interleukin-6 (IL-6) ELISA Kit (Elabscience, Shanghai, China), Human interleukin-8 (IL-8) ELISA Kit (Elabscience, Shanghai, China), and Human VEGF ELISA Kit (Mlbio, Shanghai, China).

\section{Quantitative reverse transcription-PCR (qRT-PCR)}

Total RNA was extracted by TRIZOL (Invitrogen, Carlsbad, California, USA) from the tissues and cells. Firststrand cDNA was synthesized using the Strand cDNA 
Synthesis Kit (Thermo, Waltham, MA, USA) as per the manufacturer's instructions. The qRT-PCR assays were carried out using the SYBR Real-time PCR I kit (Takara, Biotechnology Co., Ltd., Japan). The standard control for mRNA was glyceraldehyde-3-phosphate dehydrogenase (GAPDH). Quantitative determination of the RNA levels was conducted by the SYBR GreenPremix Ex TaqTM II Kit (TaKaRa, Biotechnology Co., Ltd., Japan). The experiments were independently repeated at least three times. The primer sequences are as follows:

HIF-1 $\alpha$ forward: 5'-AGAGCAGGAAAAGGAGTC-3';

$H I F-1 \alpha$ reverse:

5'-TGTACGCTTGAAAAAGTGAACCA-3'; HSP70 forward: 5'-TTTCAGTGTGTCCAGTGC-3'; HSP70 reverse: 5'-TGGTTGGTCCATCTTTTT-3'; $I G F-2$ forward: 5'-AAGTCCGAGAGGGACGT-3'; IGF-2 reverse: 5'-CTTGGGTGGGTAGAGCA-3'; IL-6 forward: 5'-TTCGGTCCAGTTGCCTTCT-3'; IL-6 reverse: 5'-CTGCCAGTGCCTCTTTGCT-3'; IL-8 forward: 5'-ATACTCCAAACCTTTCCACC-3'; IL-8 reverse: 5'-TCAAAAACTTCTCCACAACC-3'; VEGF forward: 5'-AAACTTTTCGTCCAACTT-3'; VEGF reverse: 5'-TCTTCCTTCTCTTCTTCC-3'; GAPDH forward:

5'-AGAAGGCTGGGGCTCATTTG-3'; GAPDH reverse:

5'-AGGGGCCATCCACAGTCTTC-3'.

\section{Western blot analysis}

Total proteins were extracted from the cells or mouse tissues with RIPA buffer (CST, Waltham, MA, USA). Protein concentrations were measured using the BCA Protein Quantification Kit (Abbkine, California, USA). Equivalent concentrations of protein were separated by SDS-PAGE (12\% polyacrylamide gels) and transferred to PVDF membranes (Millipore, Waltham, MA, USA) in the subsequent step. The membranes were blocked with $5 \%$ milk and incubated overnight at $4{ }^{\circ} \mathrm{C}$ with primary antibodies for HIF-1 $\alpha$ (Abcam, Massachusetts, USA), HSP70 (Abcam, Massachusetts, USA), IGF-2 (Abcam, Massachusetts, USA), IL-6 (Abcam, Massachusetts, USA), IL-8 (Abcam, Massachusetts, USA), VEGF (Abcam, Massachusetts, USA), and GAPDH (Abcam, Massachusetts, USA), where GAPDH served as the control. Membranes were then incubated with the corresponding secondary antibodies (Abcam, Massachusetts, USA) for 1 hour at room temperature, followed by visualization using an
Odyssey CLx Infrared Imaging System.

\section{Analysis of MAFT in vivo}

The effects of $A P R F / H I F-1 \alpha / V E G F$ on MAFT in vivo were analyzed in Balb/c nude mice (250-300 g, female, 4 weeks old, $\mathrm{n}=3)$. The $\mathrm{BALB} / \mathrm{c}$ mice were subcutaneously co-transplanted with fat, $A P R F$, and control shRNA, HIF-1 $\alpha$ shRNA, or $V E G F$ shRNA into the dorsal area. At 3 months after the operation, the transplanted fat tissues were harvested, weighed, and fixed for further analysis, and fat retention was calculated. The serum levels of $H I F-1 \alpha$, $H S P 70, I G F-2, I L-6, I L-8$, and $V E G F$ were analyzed by ELISA assays. The protein expression levels of $H I F-1 \alpha$, HSP70, IGF-2, IL-6, IL-8, and VEGF in the fat tissues of the mice were measured by Western blot analysis. Lipid accumulation was measured by Oil Red O staining. The expression of CD34 was assessed by immunohistochemical staining. Ethical approval to report this study involving animals was obtained from the Fifth Affiliated Hospital of Guangxi Medical University (2018-058-01), in compliance with the Fifth Affiliated Hospital of Guangxi Medical University guidelines for the care and use of animals.

\section{Statistical analysis}

Data were presented as mean $\pm \mathrm{SD}$, and statistical analysis was performed using GraphPad Prism 7 (GraphPad Software, The North Parker, USA). The unpaired Student's $t$-test was used to compare two groups, and oneway ANOVA to compare multiple groups. $\mathrm{P}<0.05$ was considered as statistically significant.

\section{Results}

\section{APRF continuously secretes multiple cytokines}

A diagram of the $A P R F$ preparation process is shown in Figure 1. To assess the potential effect of APRF on the paracrine function of ASCs, the cytokines secreted from $A P R F$ were measured by ELISA assays. We found that $A P R F$ continuously secreted multiple cytokines, including EGF (Figure 2A), FGF-2 (Figure 2B), IGF-1 (Figure 2C), IL-4 (Figure 2D), PDGF-AB (Figure 2E), PDGF-BB (Figure 2F), and $V E G F$ (Figure 2G), where day 7 showed the highest secretion. Moreover, CCK-8 assays showed that APRF treatment promoted the proliferation of ASCs in a dosedependent manner (Figure 2H). 


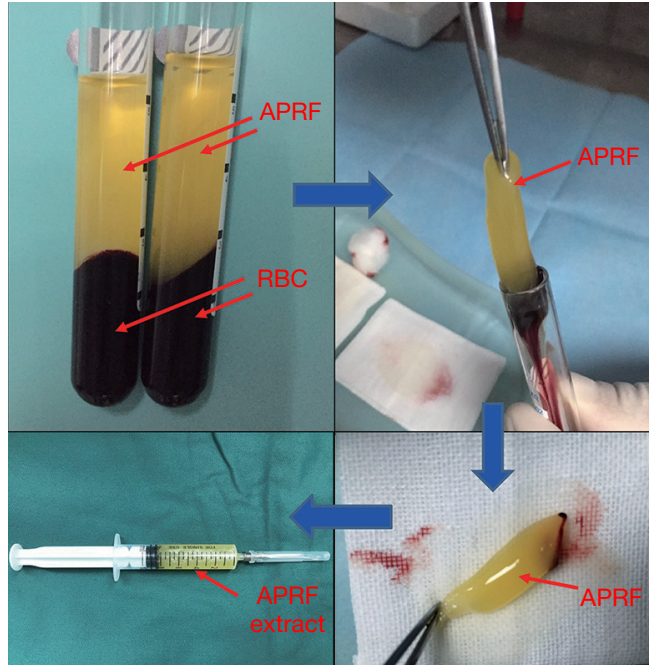

Figure 1 Diagram of the preparation process of APRF. APRF, advanced platelet-rich fibrin.

\section{APRF promotes the paracrine function of ASCs}

It has been identified that HIF signalling is involved in the modulation of ASCs (26). Accordingly, we explored the effect of $A P R F$ on HIF signalling in ASCs. ELISA assays revealed that $A P R F$ treatment dose-dependently enhanced the levels of $H I F-1 \alpha$ in the culture medium of ASCs (Figure 3A). Furthermore, the level of HSP70 in the culture medium of ASCs was increased by $A P R F$ treatment in a dose-dependent manner (Figure 3B). APRF treatment was also able to induce the levels of $I G F-2, I L-6, I L-8$, and $V E G F$ in a dose-dependent manner in the culture medium of ASCs (Figure 3C-3F). Moreover, qRT-PCR assays showed that $A P R F$ treatment dose-dependently promoted

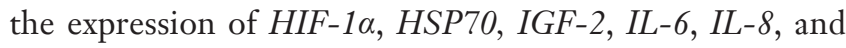
$V E G F$ in ASCs (Figure 3G-3L). Similarly, Western blot

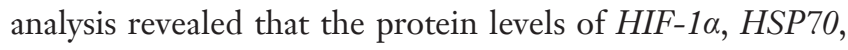
$I G F-2, I L-6, I L-8$, and $V E G F$ in ASCs were up-regulated by $A P R F$ in a dose-dependent manner (Figure $3 M-3 R$ ). Together, these data suggest that $A P R F$ can promote the paracrine function of ASCs.

\section{APRF regulates the paracrine function of ASCs by modulating HIF-1 $\alpha$ and VEGF}

We further investigated the role of $H I F-1 \alpha$ and $V E G F$ in the modulation of ASCs. To this end, ASCs were treated with lentivirus vectors carrying the shRNA of $H I F-1 \alpha$ and $V E G F$. The efficiency of shRNAs of $H I F-1 \alpha$ and $V E G F$ was validated in ASCs, and shRNA-1 of HIF-1 $\alpha$ and shRNA-2 of $V E G F$ presented more obvious effects and were therefore used in the subsequent experiments (Figure 4A,4B). ELISA assays showed that $A P R F$ treatment enhanced the levels of $H I F-1 \alpha, H S P 70, I G F-2, I L-6, I L-8$, and VEGF in the culture medium of ASCs, while depletion of $H I F-1 \alpha$ or $V E G F$ was able to reverse this enhancement (Figure $4 C-4 H$ ). Moreover, depletion of $H I F-1 \alpha$ or $V E G F$ blocked the $A P R F$-increased mRNA expression of HIF-1 $\alpha, H S P 70, I G F-2, I L-6, I L-8$, and $V E G F$ in ASCs (Figure 4I-4N). Similarly, the protein expression of $H I F-1 \alpha, H S P 70, I G F-2, I L-6, I L-8$, and $V E G F$ induced by $A P R F$ treatment was reduced by $H I F-1 \alpha$ or $V E G F$ depletion in ASCs (Figure 4O-4T). Together, these findings indicate that $A P R F$ regulates the paracrine function of ASCs by modulating HIF-1 $\alpha$ and $V E G F$.

\section{APRF contributes to MAFT in vivo}

Next, we aimed to explore the effect of APRF on MAFT in vivo. To this end, the BALB/c mice were transplanted with fat, co-transplanted with fat and ASCs or $A P R F$, or co-transplanted with fat, ASCs, and APRF. We found that $A P R F$ treatment enhanced fat retention in vivo after transplantation (Figure $5 A, 5 B$ ). Moreover, $A P R F$ treatment was able to up-regulate the serum levels of $H I F-1 \alpha$, HSP70, IGF-2, IL-6, IL-8, and VEGF (Figure 5C-5H). Similarly, the protein expression levels of HIF-1 $\alpha$, HSP70, $I G F-2, I L-6, I L-8$, and $V E G F$ in the fat tissues of the mice were increased by APRF treatment (Figure $5 I-5 N$ ). Furthermore, Oil Red $\mathrm{O}$ staining revealed that $A P R F$ could promote lipid accumulation in the mice (Figure 50 , $\times 100)$. Immunohistochemical staining revealed that $A P R F$ treatment was able to enhance the expression of CD34 in the fat tissues of the mice (Figure 5P, $\times 100$ ). Together, these data suggest that $A P R F$ contributes to MAFT in vivo.

\section{APRF promotes MAFT by modulating HIF-1 $\alpha$ and VEGF in vivo}

We further explored whether HIF-1 $\alpha$ and $V E G F$ were involved in $A P R F$-mediated MAFT in vivo. To this end, the BALB/c mice were co-transplanted with fat, $A P R F$, and control shRNA, HIF-1 $\alpha$ shRNA, or VEGF shRNA. Remarkably, depletion of $H I F-1 \alpha$ or $V E G F$ could attenuate $A P R F$ treatment-enhanced fat retention in vivo after transplantation (Figure 6A,6B). Moreover, $H I F-1 \alpha$ or $V E G F$ knockdown could reduce the $A P R F$-induced levels of $H I F-1 \alpha, H S P 70, I G F-2, I L-6, I L-8$, and $V E G F$ in the 

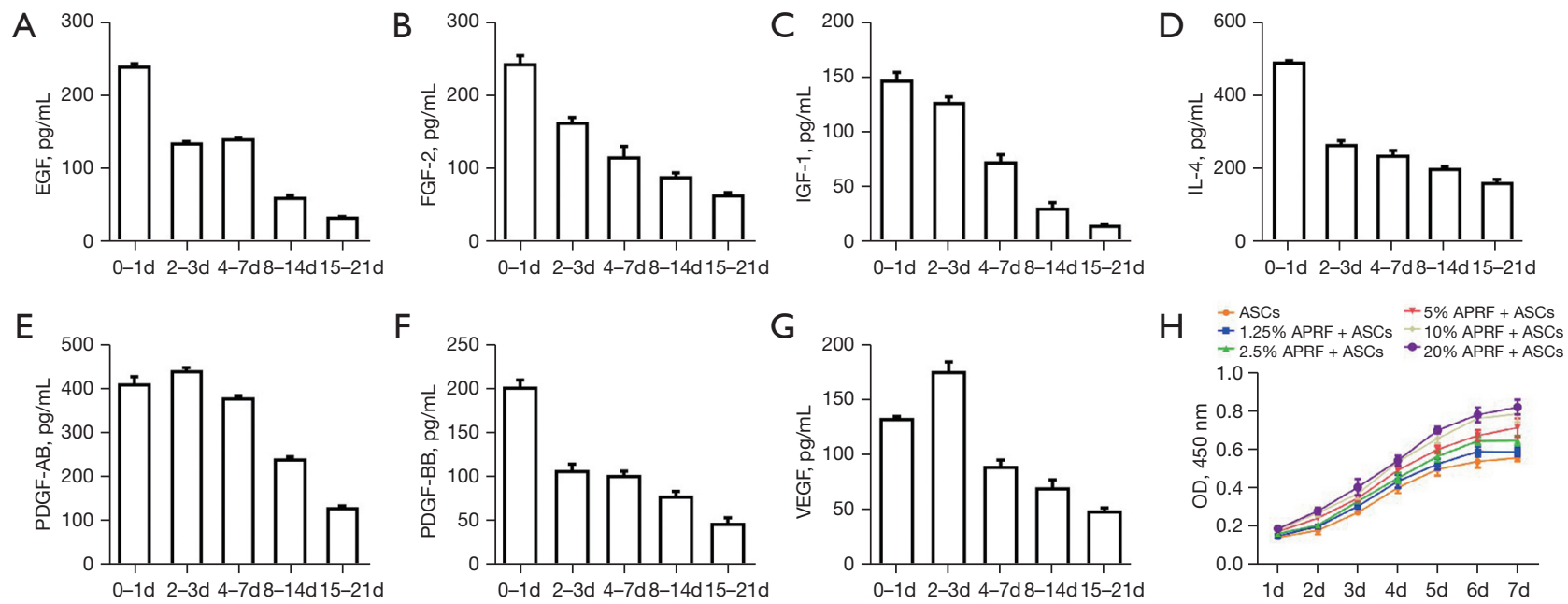

Figure 2 APRF continuously secretes multiple cytokines. The secretion of $E G F$ (A), $F G F-2$ (B), IGF-1 (C), IL-4 (D), $P D G F-A B$ (E), $P D G F-B B(\mathrm{~F})$, and $\operatorname{VEGF}(\mathrm{G})$ from $A P R F$ was analyzed by ELISA assays. (H) ASCs were treated with $A P R F$ at the indicated dose. The cell proliferation of ASCs was analyzed by CCK-8 assays at the indicated time. APRF, advanced platelet-rich fibrin; EGF, epidermal growth factor; $I G F$-1, insulin-like growth factor-1; $P D G F$, platelet-derived growth factor; $T G F-\beta$, transforming growth factor-beta; VEGF, vascular endothelial growth factor; ASCs, adipose-derived stem cells.

serum of the mice (Figure $6 \mathrm{C}-6 \mathrm{H}$ ). Similarly, the protein expression levels of $H I F-1 \alpha, H S P 70, I G F-2, I L-6, I L-8$, and $V E G F$ in the fat tissues of the mice increased by $A P R F$ treatment were inhibited by $H I F-1 \alpha$ or $V E G F$ depletion (Figure 6I-6N). Furthermore, Oil Red O staining revealed that $H I F-1 \alpha$ or VEGF depletion could decrease APRFenhanced lipid accumulation in the mice (Figure $6 O, \times 100$ ). Immunohistochemical staining revealed that the $A P R F$ increased expression of CD34 in the fat tissues of the mice was blocked by depletion of HIF-1 $\alpha$ or VEGF (Figure 6P, $\times 100$ ). Together, these data suggest that $A P R F$ promotes the paracrine function in vivo and contributes to MAFT by modulating HIF-1 $\alpha$ and $V E G F$.

\section{Discussion}

As an autologous platelet concentrate, $A P R F$ has been demonstrated to play a role in the modulation of fat grafting. It has been reported that $A P R F$ increases survival after fat grafting by regulating collagen production, inhibiting apoptosis, and promoting adipogenesis and angiogenesis (27). $A P R F$ and platelet-rich plasma improve fat graft outcomes (28). Nano fat-derived stem cells with $A P R F$ enhance skin rejuvenation and facial shape improvement following structural autologous fat transplantation (29). Autogenous-free fat grafting combined with $A P R F$ recovers firm mandibular osteoradionecrosis (30). APRF and the adjuvant stromal vascular fraction (SVF) application for autologous fat tissue transplantation have been reported (31). In this study, we firstly identified that $A P R F$ promoted the paracrine function and proliferation of ASCs. We also found that $A P R F$ contributed to MAFT in vivo. These data present a novel function of $A P R F$ in the modulation of MAFT, providing valuable evidence for the fundamental role of $A P R F$ in fat grafting.

Furthermore, it has been identified that HIF signaling is involved in the modulation of fat grafting. $\mathrm{CD} 54^{+}$rabbit ASCs overexpressing $H I F-1 \alpha$ enhanced vascularized fat flap regeneration (21). The $\mathrm{Wnt} / \beta$-catenin and $H I F-1 \alpha$ signalling pathways were shown to be involved in the protective effect of losartan on fatty liver graft with ischemia/reperfusion injury (32). Hypoxia improves the

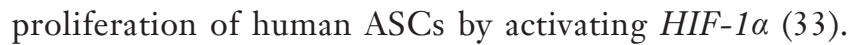
Moreover, it has been reported that SVF cells combined with sustained-release VEGF/Ang-1-PLGA microspheres enhance the survival of fat grafts in mice (34). Recipientsite preconditioning with deferoxamine promotes fat graft survival through stimulating neovascularization and $V E G F$ in vivo (35). VEGF-transfected ASCs improved the survival of autologous fat transplantation (36). VEGF-PLA nanosustained release microspheres and SVF enhanced the survival of autologous human fat transplantation (37). Our 

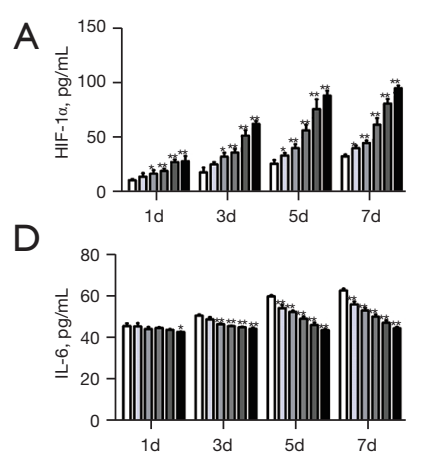

G
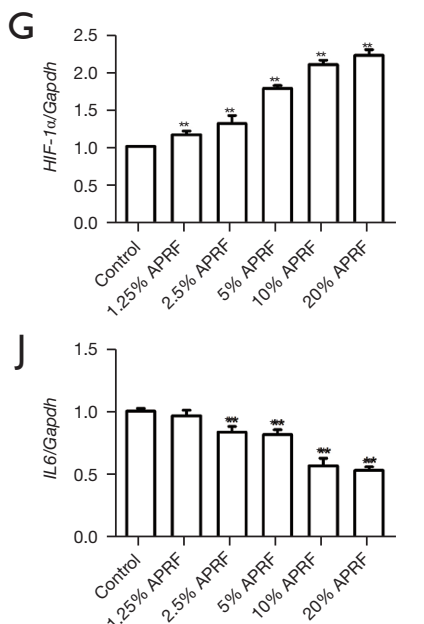

M

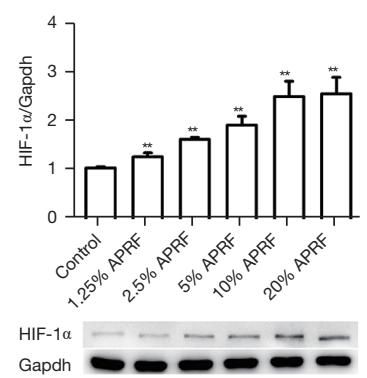

$\mathrm{P}$

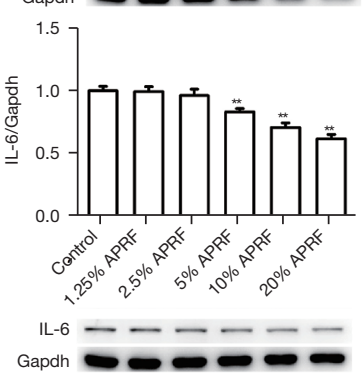

$\mathrm{B}$

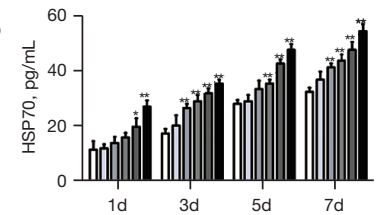

E

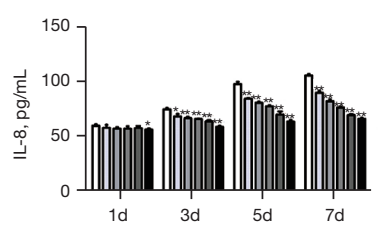

$\mathrm{H}$
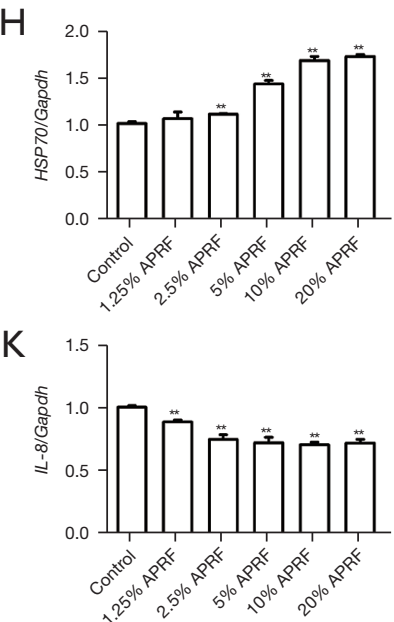

$\mathrm{N}$
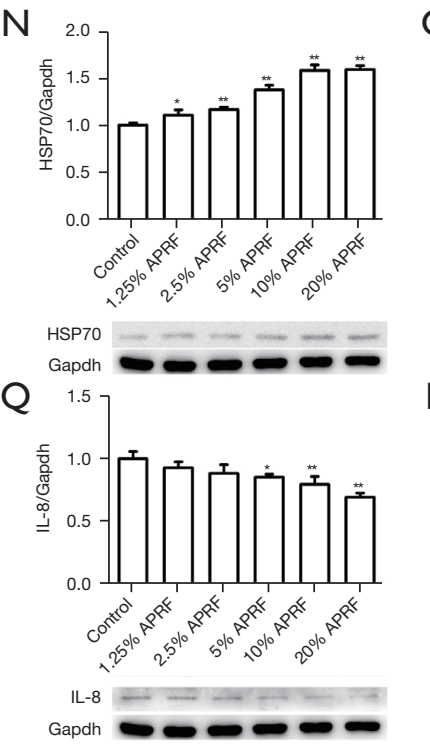

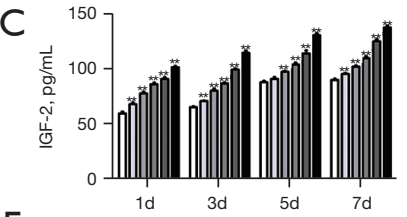

F

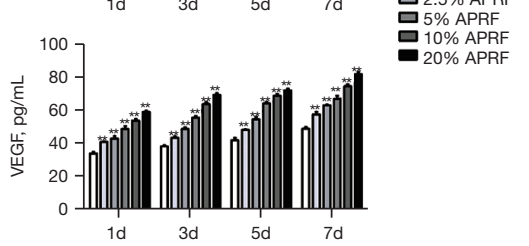

I

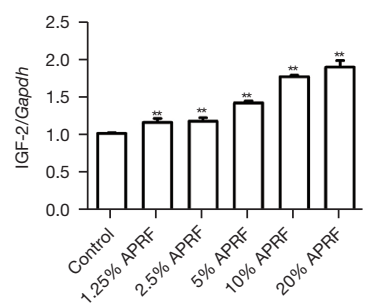

$\mathrm{L}$

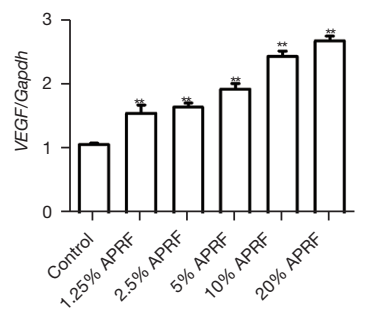

O

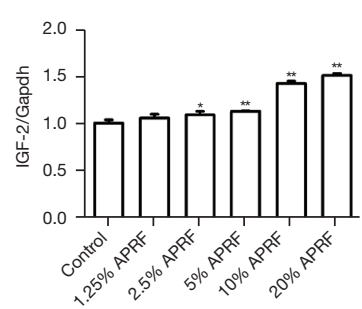

$\mathrm{R}$

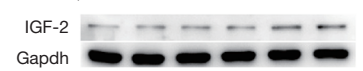

R

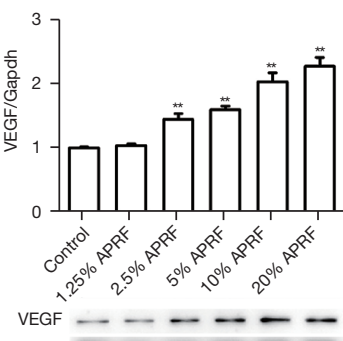

Figure 3 APRF promotes the paracrine function of ASCs. (A-R) ASCs were treated with APRF at the indicated dose. (A-F) The levels of HIF-1 $\alpha, H S P 70, I G F-2, I L-6, I L-8$, and $V E G F$ in the culture medium of ASCs were analyzed by ELISA assays at the indicated dose. (G-L) The mRNA expression levels of HIF-1 $\alpha$, HSP70, IGF-2, IL-6, IL-8, and VEGF in ASCs were measured by qRT-PCR assays. (M-R) The protein expression levels of HIF-1 $\alpha, H S P 70, I G F-2, I L-6, I L-8$, and VEGF in ASCs were measured by Western blot analysis. The results of Western blot analysis were quantified by ImageJ software. Data are presented as mean \pm SD. Significant differences are indicated by *, $\mathrm{P}<0.05$; ** $\mathrm{P}<0.01$. APRF, advanced platelet-rich fibrin; ASCs, adipose-derived stem cells; HIF-1 $\alpha$, hypoxia-inducible factor-1 $\alpha$; VEGF, vascular endothelial growth factor; qRT-PCR, quantitative reverse transcription-PCR. 


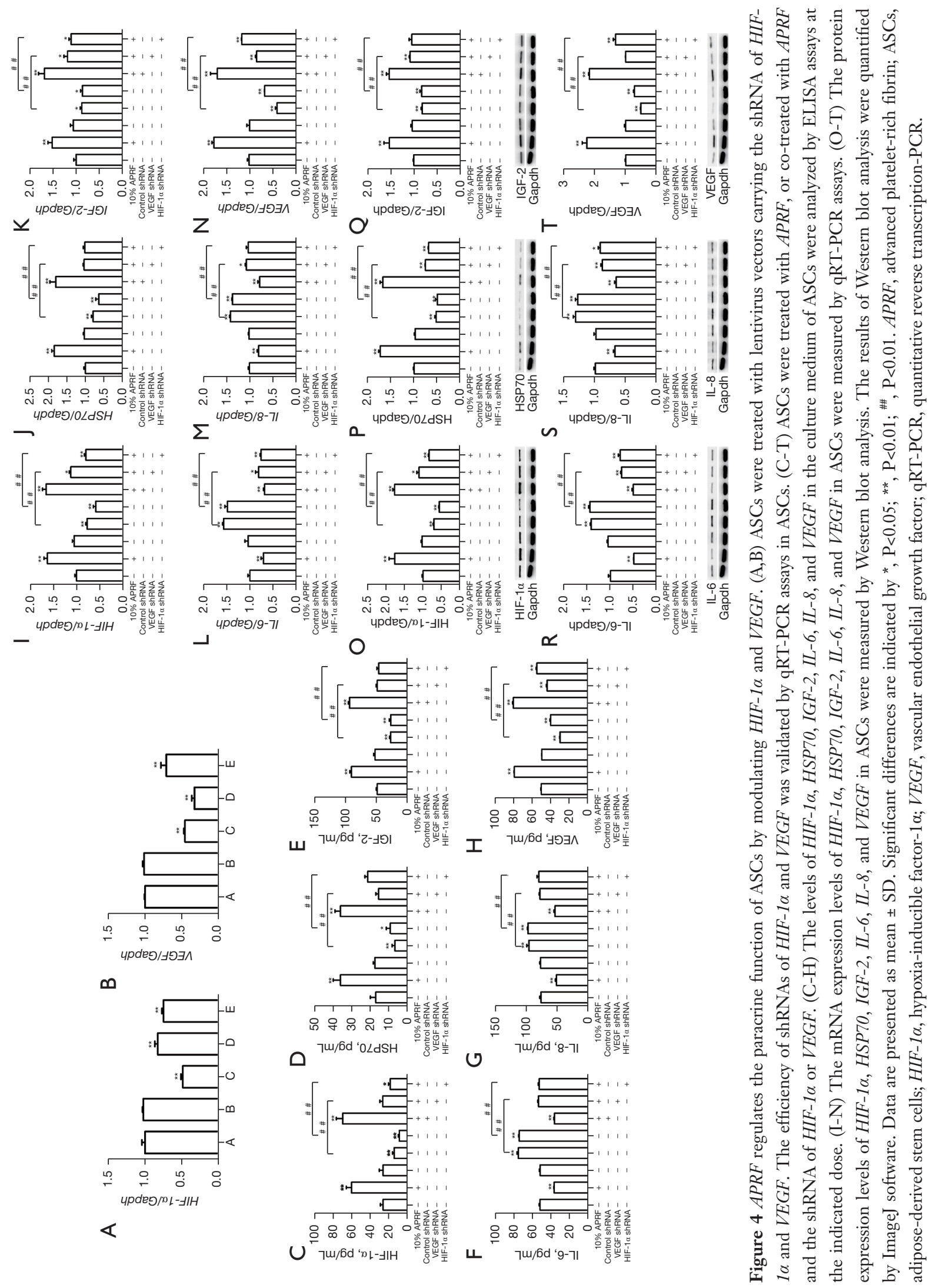


A

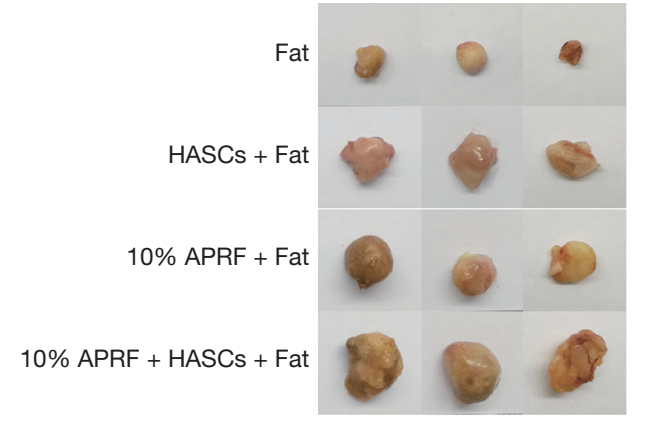

D 150 E

$\mathrm{F}$

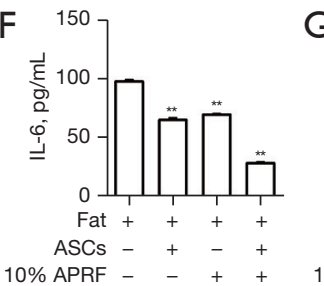

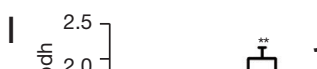
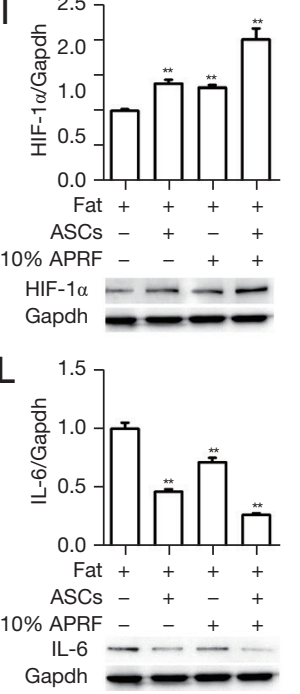

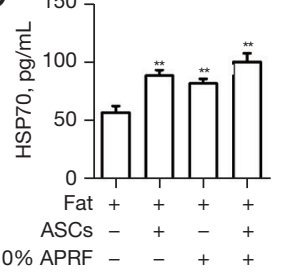

E
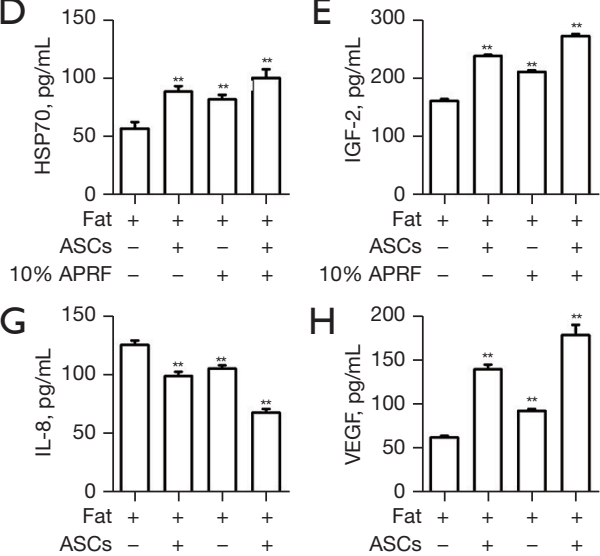

$10 \%$ APRF $-\quad+\quad++$
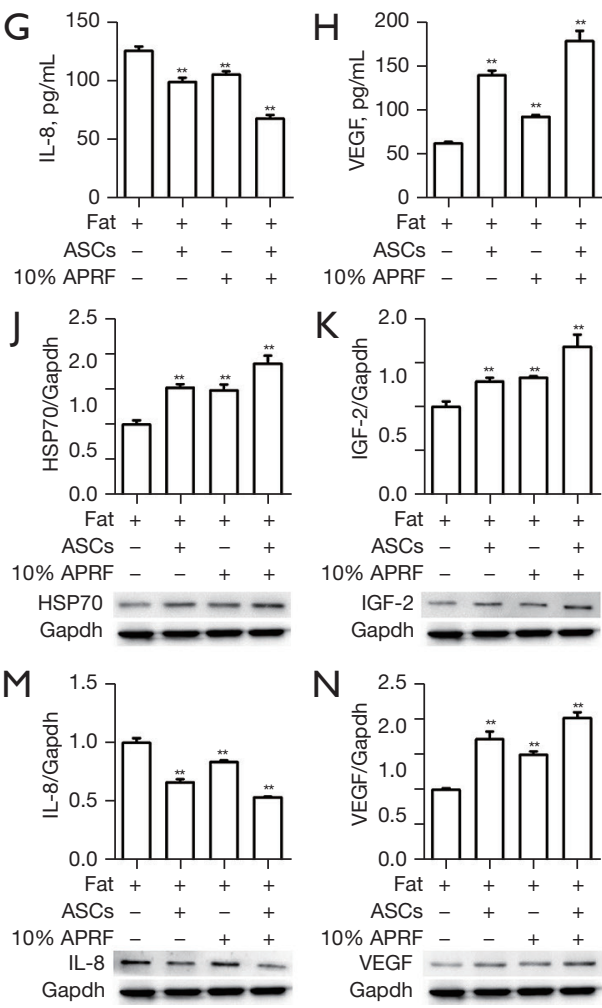

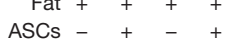

$10 \%$ APRF -++

N

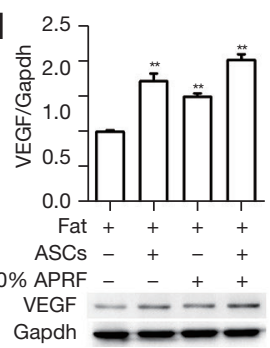

B
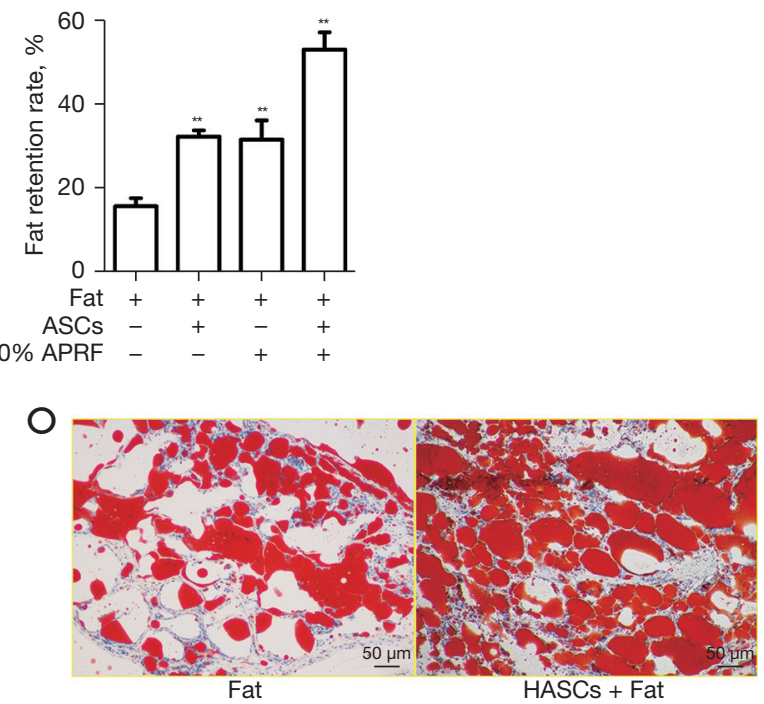

$\mathrm{K}^{2.0}$

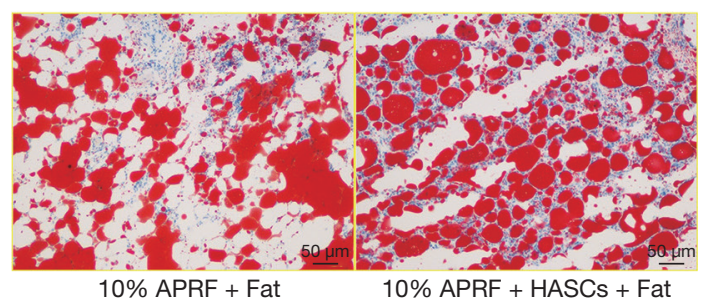

$\mathrm{P}$
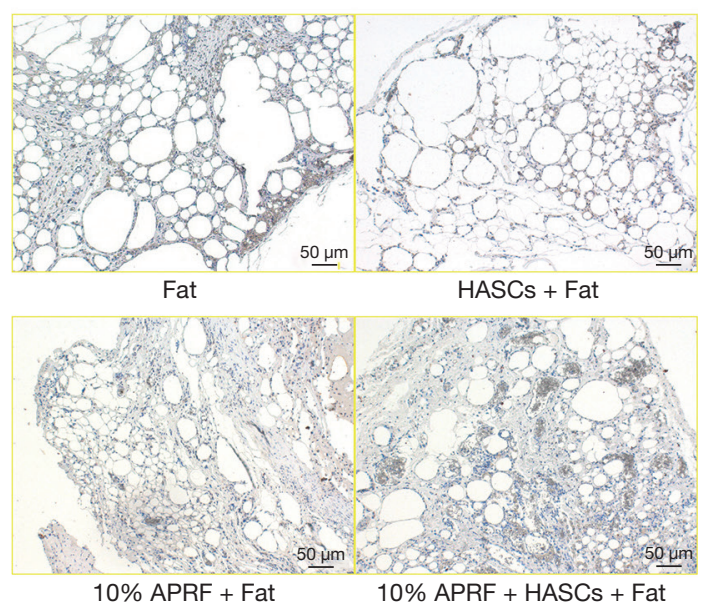

Figure 5 APRF contributes to micro-autologous fat transplantation in vivo. (A-P) BALB/c mice ( $\mathrm{n}=3$ ) were transplanted with fat, cotransplanted with fat and ASCs or APRF, or co-transplanted with fat, ASCs, and APRF as indicated. (A) Representative images of transplanted fat in mice. (B) The fat retention rate was calculated. (C-H) The serum levels of HIF-1 $\alpha, H S P 70, I G F-2, I L-6, I L-8$, and $V E G F$ were analyzed by ELISA assays. (I-N) The protein expression levels of HIF-1 $\alpha, H S P 70, I G F-2, I L-6, I L-8$, and $V E G F$ in the fat tissues of the mice were measured by Western blot analysis. The results of Western blot analysis were quantified by ImageJ software. (O) Lipid accumulation was measured by Oil Red O staining. (P) The expression of CD34 was assessed by immunohistochemical staining. Data are presented as mean $\pm \mathrm{SD}$. Significant differences are indicated by ${ }^{* *}, \mathrm{P}<0.01$. APRF, advanced platelet-rich fibrin; ASCs, adipose-derived stem cells; HIF-1 $\alpha$, hypoxia-inducible factor-1 $\alpha$; VEGF, vascular endothelial growth factor. 

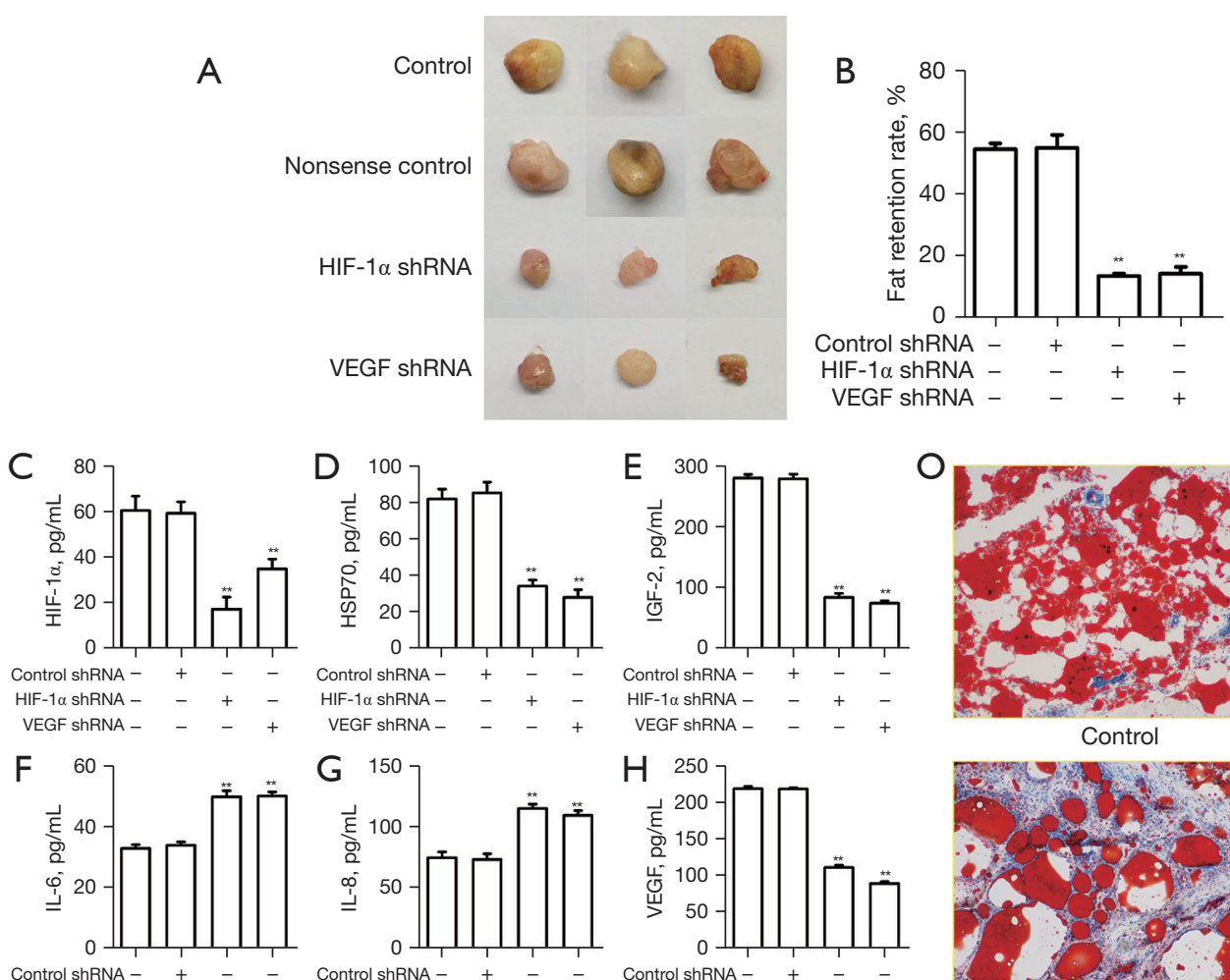

HIF-1 1 ShRNA

VEGF ShRNA - $-\quad-+$

VEGF ShRNA
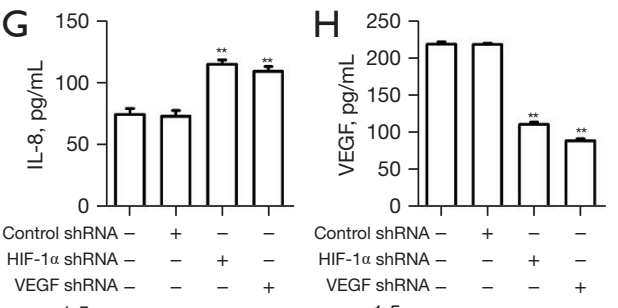

$\begin{array}{llll}\text { HIF- } 1 \alpha \text { shRNA } & - & + & - \\ \text { VEGF shRNA } & - & - & + \\ \text { | } 1.5\end{array}$
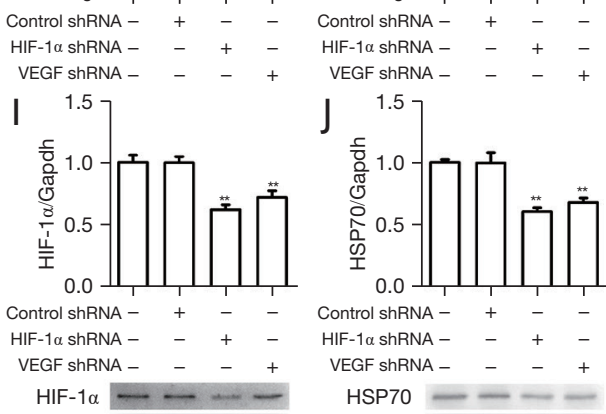

VEGF ShRNA - $\quad-\quad$

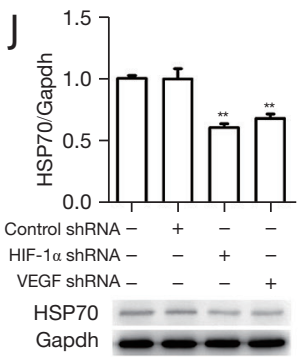

$\mathrm{K}$
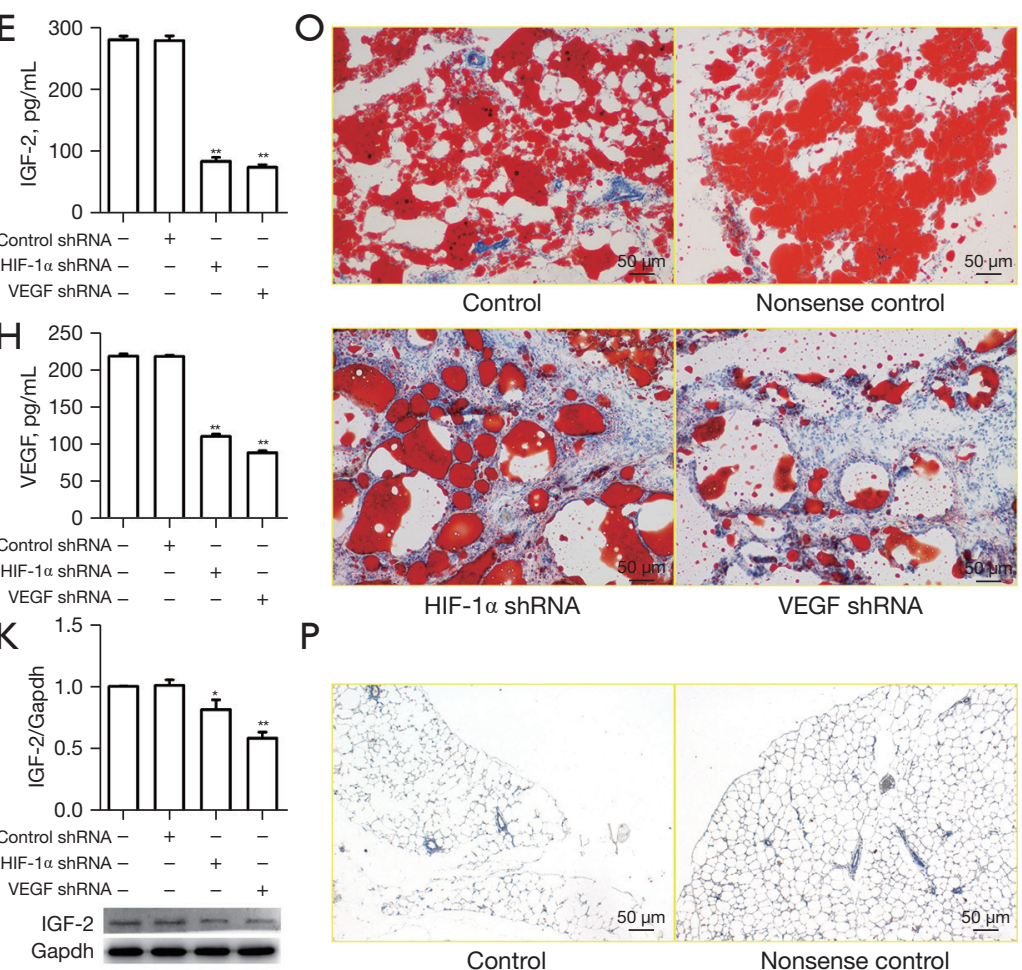

Gapdh $\longrightarrow$
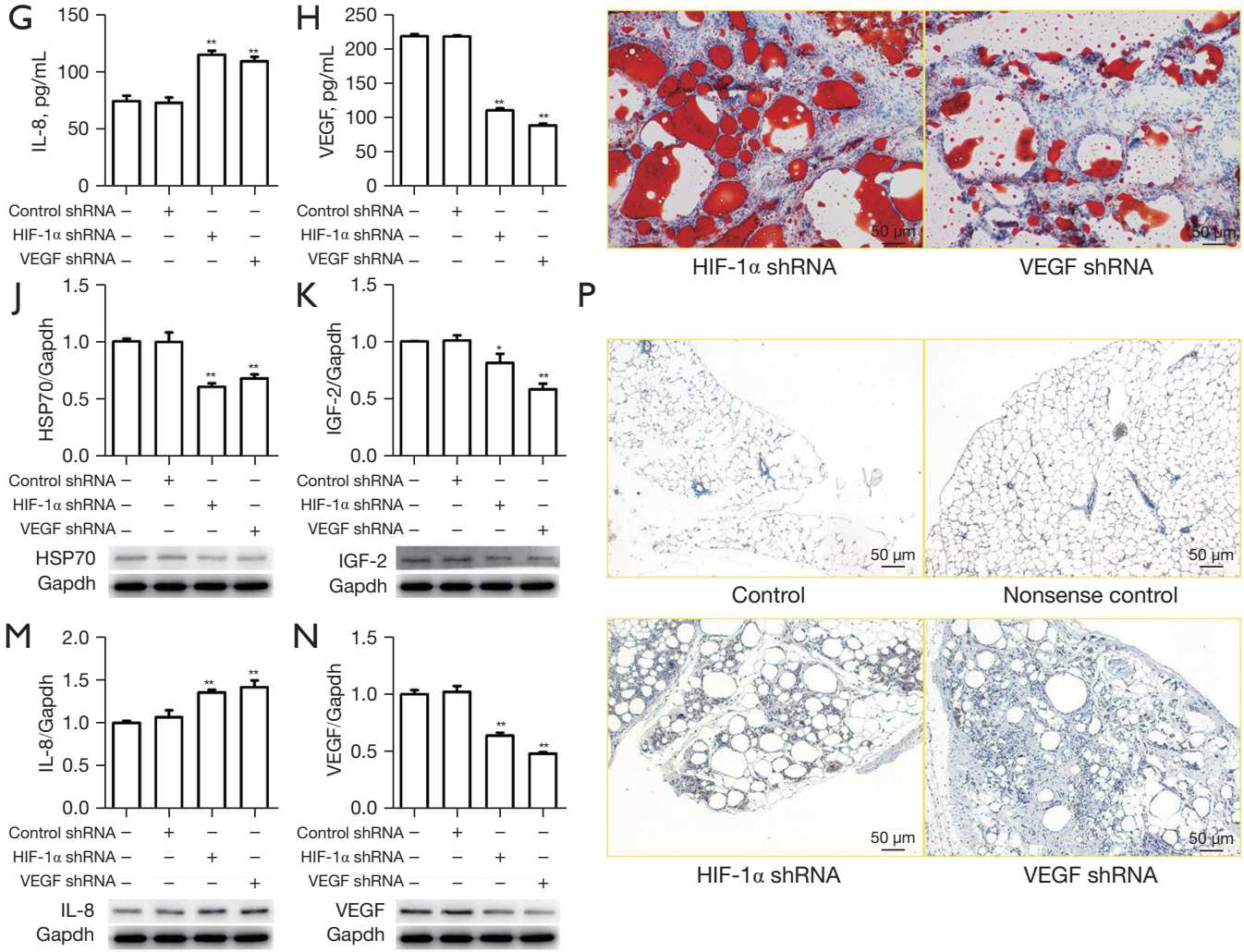

$P$
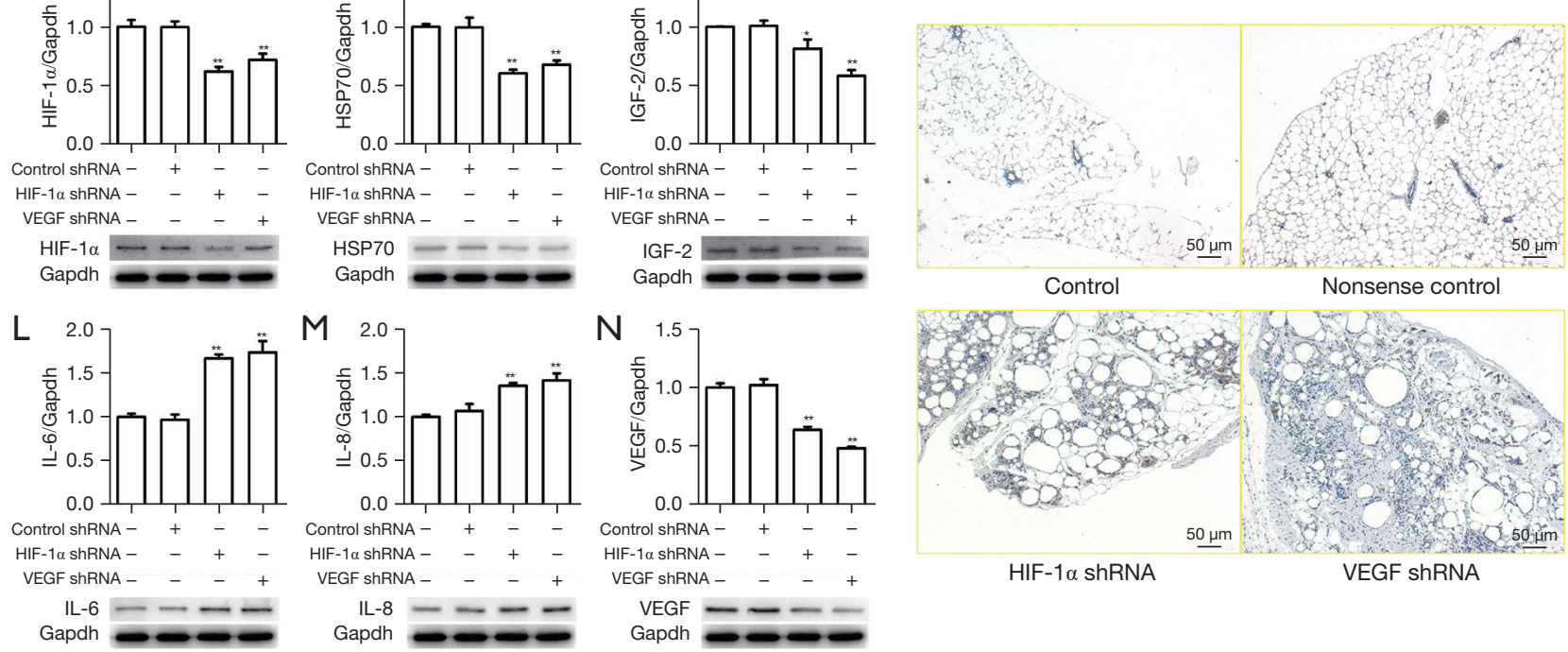

Figure 6 APRF promotes micro-autologous fat transplantation by modulating HIF-1 $\alpha$ and $V E G F$ in vivo. (A-P) BALB/c mice (n=3) were co-transplanted with fat, ASCs, $A P R F$, and control shRNA, HIF-1 $\alpha$ shRNA, or $V E G F$ shRNA. (A,B) Representative images of transplanted fat in the mice. (B) The fat retention rate was calculated. (C-H) The serum levels of HIF-1 $\alpha, H S P 70, I G F-2, I L-6, I L-8$, and VEGF were analyzed by ELISA assays. (I-N) The protein expression levels of HIF-1 $\alpha$, HSP70, IGF-2, IL-6, IL-8, and VEGF in the fat tissues of the mice were measured by Western blot analysis. The results of Western blot analysis were quantified by ImageJ software. (O) Lipid accumulation was measured by Oil Red O staining. (P) The expression of CD34 was assessed by immunohistochemical staining. Data are presented as mean $\pm \mathrm{SD}$. Significant differences are indicated by *, $\mathrm{P}<0.05 ;{ }^{* *}, \mathrm{P}<0.01$. APRF, advanced platelet-rich fibrin; HIF-1 $\alpha$, hypoxia-inducible factor-1 $\alpha$; $V E G F$, vascular endothelial growth factor. 
mechanical investigation further demonstrated that $A P R F$ regulated the paracrine function of ASCs by modulating $H I F-1 \alpha$ and $V E G F$. APRF promoted MAFT by modulating $H I F-1 \alpha$ and $V E G F$ in vivo. These data highlight an unreported role of $H I F-1 \alpha$ and $V E G F$ in $A P R F$-mediated ASC paracrine function and fat transplantation, identifying a new correlation of $A P R F$ with $H I F-1 \alpha$ and VEGF.

Adipocytes secrete more than 100 factors, contributing to the expression of growth factors and cytokines such as adiponectin, IGF, fibroblast growth factor (FGF), and $V E G F$ (38). PDGF, $F G F-2$, and $V E G F$ are tyrosine kinase receptor-mediated growth factors that have been shown to improve fat graft transplantation (38). It has been reported that MAFT is correlated with platelet-rich plasma (PRP), which includes multiple angiogenic growth factors such as $F G F, V E G F, E G F, T G F-\beta$, and PDGF (39). This technique has been shown to increase the proliferation of adipocytederived stem cells and enhance overall graft survival in vitro and in vivo (39). Our data showed that $A P R F$ induced the secretion of $E G F, F G F-2, I G F-1, I L-1 \beta, I L-4, P D G F-A B$, $P D G F-B B, T G F-\beta$, and $V E G F$ in the culture medium of ASCs. These data provide new evidence that these factors are crucial for $A P R F$-regulated paracrine functioning.

In conclusion, we discovered that $A P R F$ promoted the paracrine function and proliferation of ASCs, and contributed to MAFT by modulating HIF-1 $\alpha$ and $V E G F$. Our findings provide new insights into the mechanism by which $A P R F$ regulates MAFT.

\section{Acknowledgments}

Funding: This work was financially supported by the National Nature Science Foundation of China (81760346), the Guangxi Natural Science Foundation (2018GXNSFAA281148), the Scientific Research \& Technology Development Program of Nanning (20183037-1, 20191034), the Yong River Program of Innovation and Entrepreneurship of Nanning (2018-01-07), and the Youth Science Innovation and Entrepreneurship Talent Training Project of Nanning (RC20190206).

\section{Footnote}

Reporting Checklist: The authors have completed the ARRIVE reporting checklist. Available at https://atm. amegroups.com/article/view/10.21037/atm-21-6812/rc

Data Sharing Statement: Available at https://atm.amegroups. com/article/view/10.21037/atm-21-6812/dss

Conflicts of Interest: All authors have completed the ICMJE uniform disclosure form (available at https://atm. amegroups.com/article/view/10.21037/atm-21-6812/ coif). HL reports that this work was financially supported by the National Nature Science Foundation of China (81760346), the Guangxi Natural Science Foundation (2018GXNSFAA281148), the Scientific Research \& Technology Development Program of Nanning (201830371, 20191034), the Yong River Program of innovation and entrepreneurship of Nanning (2018-01-07), the Youth Science Innovation and Entrepreneurship Talent Training Project of Nanning (RC20190206), of which the authors are greatly appreciative. DL and FW are from Nanning Wilking Biological Technology Co., Ltd. The other authors have no conflicts of interest to declare.

Ethical Statement: The authors are accountable for all aspects of the work in ensuring that questions related to the accuracy or integrity of any part of the work are appropriately investigated and resolved. The study was conducted in accordance with the Declaration of Helsinki (as revised in 2013). The present study was approved by the Institutional Review Board of the Fifth Affiliated Hospital of Guangxi Medical University (Nanning, Guangxi, China) (No. 2019-107-01). Written informed consent was provided by the donor patients. Ethical approval to report this study involving animals was obtained from the Fifth Affiliated Hospital of Guangxi Medical University (No. 2018-058-01), in compliance with the Fifth Affiliated Hospital of Guangxi Medical University guidelines for the care and use of animals.

Open Access Statement: This is an Open Access article distributed in accordance with the Creative Commons Attribution-NonCommercial-NoDerivs 4.0 International License (CC BY-NC-ND 4.0), which permits the noncommercial replication and distribution of the article with the strict proviso that no changes or edits are made and the original work is properly cited (including links to both the formal publication through the relevant DOI and the license). See: https://creativecommons.org/licenses/by-nc-nd/4.0/.

\section{References}

1. Ozer K, Colak O. Micro-Autologous Fat Transplantation Combined With Platelet-Rich Plasma for Facial Filling 
and Regeneration: A Clinical Perspective in the Shadow of Evidence-Based Medicine. J Craniofac Surg 2019;30:672-7.

2. Pu LLQ. Fat Grafting for Facial Rejuvenation and Contouring: A Rationalized Approach. Ann Plast Surg 2018;81:S102-8.

3. Coleman SR, Katzel EB. Fat Grafting for Facial Filling and Regeneration. Clin Plast Surg 2015;42:289-300, vii.

4. Huang SH, Lin YN, Lee SS, et al. Three Simple Steps for Refining Transcutaneous Lower Blepharoplasty for Aging Eyelids: The Indispensability of Micro-Autologous Fat Transplantation. Aesthet Surg J 2019;39:1163-77.

5. Strong AL, Cederna PS, Rubin JP, et al. The Current State of Fat Grafting: A Review of Harvesting, Processing, and Injection Techniques. Plast Reconstr Surg 2015;136:897-912.

6. Chou CK, Lee SS, Lin TY, et al. Micro-autologous Fat Transplantation (MAFT) for Forehead Volumizing and Contouring. Aesthetic Plast Surg 2017;41:845-55.

7. Yun-Nan L, Shu-Hung H, Tsung-Ying L, et al. Microautologous fat transplantation for rejuvenation of the dorsal surface of the aging hand. J Plast Reconstr Aesthet Surg 2018;71:573-84.

8. Lin YN, Chuang CH, Huang SH, et al. Fat grafting for resurfacing an exposed implant in lower extremity: A case report. Medicine (Baltimore) 2017;96:e8901.

9. Czerniak S, Gusenoff JA, Peter Rubin J. Commentary: Micro-Autologous Fat Transplantation (MAFT) for Forehead Volumizing and Contouring. Aesthetic Plast Surg 2017;41:1093-5.

10. Lee SS, Huang YH, Lin TY, et al. Long-Term Outcome of Microautologous Fat Transplantation to Correct Temporal Depression. J Craniofac Surg 2017;28:629-34.

11. Kao WP, Lin YN, Lin TY, et al. Microautologous Fat Transplantation for Primary Augmentation Rhinoplasty: Long-Term Monitoring of 198 Asian Patients. Aesthet Surg J 2016;36:648-56.

12. Lin TM, Lin TY, Huang YH, et al. Fat Grafting for Recontouring Sunken Upper Eyelids With Multiple Folds in Asians-Novel Mechanism for Neoformation of Double Eyelid Crease. Ann Plast Surg 2016;76:371-5.

13. Tang XB, Dong PL, Wang J, et al. Effect of autologous platelet-rich plasma on the chondrogenic differentiation of rabbit adipose-derived stem cells in vitro. Exp Ther Med 2015;10:477-83.

14. Miron RJ, Pinto NR, Quirynen M, et al. Standardization of relative centrifugal forces in studies related to plateletrich fibrin. J Periodontol 2019;90:817-20.

15. Kobayashi E, Flückiger L, Fujioka-Kobayashi M, et al.
Comparative release of growth factors from PRP, PRF, and advanced-PRF. Clin Oral Investig 2016;20:2353-60.

16. Li Q, Reed DA, Min L, et al. Lyophilized platelet-rich fibrin (PRF) promotes craniofacial bone regeneration through Runx2. Int J Mol Sci 2014;15:8509-25.

17. Lucarelli E, Beretta R, Dozza B, et al. A recently developed bifacial platelet-rich fibrin matrix. Eur Cell Mater 2010;20:13-23.

18. Dohan DM, Choukroun J, Diss A, et al. Platelet-rich fibrin (PRF): a second-generation platelet concentrate. Part II: platelet-related biologic features. Oral Surg Oral Med Oral Pathol Oral Radiol Endod 2006;101:e45-50.

19. Harris AL. Hypoxia---a key regulatory factor in tumour growth. Nat Rev Cancer 2002;2:38-47.

20. Semenza GL. Oxygen sensing, hypoxia-inducible factors, and disease pathophysiology. Annu Rev Pathol 2014;9:47-71.

21. Li DQ, Lu GM, Liang YD, et al. CD54+ rabbit adipose-derived stem cells overexpressing HIF-1 $\alpha$ facilitate vascularized fat flap regeneration. Oncotarget 2017;8:46875-90.

22. Conley SM, Hickson LJ, Kellogg TA, et al. Human Obesity Induces Dysfunction and Early Senescence in Adipose Tissue-Derived Mesenchymal Stromal/Stem Cells. Front Cell Dev Biol 2020;8:197.

23. Kakudo N, Shimotsuma A, Kusumoto K. Fibroblast growth factor-2 stimulates adipogenic differentiation of human adipose-derived stem cells. Biochem Biophys Res Commun 2007;359:239-44.

24. Kakudo N, Minakata T, Mitsui T, et al. Proliferationpromoting effect of platelet-rich plasma on human adipose-derived stem cells and human dermal fibroblasts. Plast Reconstr Surg 2008;122:1352-60.

25. Kakudo N, Kushida S, Suzuki K, et al. Effect of C3 transferase on human adipose-derived stem cells. Hum Cell 2011;24:165-9.

26. Cun X, Xie J, Lin S, et al. Gene profile of soluble growth factors involved in angiogenesis, in an adipose-derived stromal cell/endothelial cell co-culture, 3D gel model. Cell Prolif 2015;48:405-12.

27. Yu P, Zhai Z, Lu H, et al. Platelet-Rich Fibrin Improves Fat Graft Survival Possibly by Promoting Angiogenesis and Adipogenesis, Inhibiting Apoptosis, and Regulating Collagen Production. Aesthet Surg J 2020;40:NP530-45.

28. Xiong S, Qiu L, Su Y, et al. Platelet-Rich Plasma and Platelet-Rich Fibrin Enhance the Outcomes of Fat Grafting: A Comparative Study. Plast Reconstr Surg 2019;143:1201e-12e. 
29. Wei H, Gu SX, Liang YD, et al. Nanofat-derived stem cells with platelet-rich fibrin improve facial contour remodeling and skin rejuvenation after autologous structural fat transplantation. Oncotarget 2017;8:68542-56.

30. Law B, Mohd Yunus SS, Ramli R. Autogenous free fat graft combined with platelet-rich fibrin heals a refractory mandibular osteoradionecrosis. Clin Ter 2020;171:e110-3.

31. Liu B, Tan XY, Liu YP, et al. The adjuvant use of stromal vascular fraction and platelet-rich fibrin for autologous adipose tissue transplantation. Tissue Eng Part C Methods 2013;19:1-14.

32. Yang YY, Lee PC, Huang YT, et al. Involvement of the HIF- $1 \alpha$ and $W n t / \beta$-catenin pathways in the protective effects of losartan on fatty liver graft with ischaemia/ reperfusion injury. Clin Sci (Lond) 2014;126:163-74.

33. Kakudo N, Morimoto N, Ogawa T, et al. Hypoxia enhances proliferation of human adipose-derived stem cells via HIF-1a activation. PLoS One 2015;10:e0139890.

34. Fan M, Li X, Gao X, et al. LPS Induces Preeclampsia-Like Phenotype in Rats and HTR8/SVneo Cells Dysfunction Through TLR4/p38 MAPK Pathway. Front Physiol

Cite this article as: Huang H, Tang X, Li S, Huang D, Lu D, Wu F, Liu D, Li H. Advanced platelet-rich fibrin promotes the paracrine function and proliferation of adipose-derived stem cells and contributes to micro-autologous fat transplantation by modulating HIF-1 $\alpha$ and VEGF. Ann Transl Med 2022;10(2):60. doi: $10.21037 / \mathrm{atm}-21-6812$
2019; 10:1030.

35. Kim J, Park M, Jeong W, et al. Recipient-Site Preconditioning with Deferoxamine Increases Fat Graft Survival by Inducing VEGF and Neovascularization in a Rat Model. Plast Reconstr Surg 2019;144:619e-29e.

36. Lu F, Li J, Gao J, et al. Improvement of the survival of human autologous fat transplantation by using VEGFtransfected adipose-derived stem cells. Plast Reconstr Surg 2009;124:1437-46.

37. Li L, Pan S, Ni B, et al. Improvement in autologous human fat transplant survival with SVF plus VEGFPLA nano-sustained release microspheres. Cell Biol Int 2014;38:962-70.

38. Diepenbrock RM, Green JM 3rd. Autologous Fat Transfer for Maxillofacial Reconstruction. Atlas Oral Maxillofac Surg Clin North Am 2018;26:59-68.

39. Liao HT, James IB, Marra KG, et al. The Effects of Platelet-Rich Plasma on Cell Proliferation and Adipogenic Potential of Adipose-Derived Stem Cells. Tissue Eng Part A 2015;21:2714-22. 